\title{
Activités
}

9-2 | Octobre 2012

Que faire du Lean?

\section{Rémi Bazillier, Le travail, grand oublié du développement durable}

Pascal Béguin

\section{OpenEdition}

1 Journals

Édition électronique

URL : http://journals.openedition.org/activites/508

DOI : 10.4000/activites.508

ISSN : 1765-2723

Éditeur

ARPACT - Association Recherches et Pratiques sur les ACTivités

Référence électronique

Pascal Béguin, « Rémi Bazillier, Le travail, grand oublié du développement durable », Activités [En ligne], 9-2 I Octobre 2012, mis en ligne le 15 octobre 2012, consulté le 23 septembre 2020. URL : http:// journals.openedition.org/activites/508; DOI : https://doi.org/10.4000/activites.508

\section{(C) $\Theta \Theta$}

Activités est mis à disposition selon les termes de la licence Creative Commons Attribution - Pas d'Utilisation Commerciale - Pas de Modification 4.0 International. 


\section{Analyse d'ouvrage par Pascale Béguin}

\section{R. Bazillier (2011). Le travail, grand oublié du développement durable. Cavalier Bleu, Coll. eDen, 213 pages}

La question du développement durable fait aujourd'hui les gros titres de la presse. Mais quelle place convient-il de donner au travail ? Voilà la (vaste) question que cherche à traiter cet ouvrage composé de deux sections. La première, titrée « comprendre et agir »; comporte 4 chapitres: (i) Le travail et la dimension sociale du développement durable, (ii) Les conditions de travail dans le monde, (iii) Travail et développement et (iv) Travail et environnement. La seconde section est titrée «acteurs », et elle dresse un très bref inventaire des acteurs et des institutions impliqués sur le versant social du développement durable.

Bazillier s'inscrit (résolument!) dans la continuité du rapport de Mme Brundtland: la dimension sociale du développement durable et en particulier le travail et l'emploi, qui sont explicitement désignés dans le rapport de Mme Brundtland au titre des besoins des individus, constituent un des piliers du développement durable.

Or cette dimension sociale du développement marque le pas sur la sphère économique. Brazillier argumente que ceci a pour origine une conception dominante : c'est la croissance (la sphère économique) qui est considérée comme étant le facteur d'amélioration du social et de l'environnemental (comme tend à l'attester la déclaration des pays du sud «notre pollution c'est la pauvreté »).

Bazillier discute cette causalité : le travail (accès à l'emploi et conditions de travail) est au cœur de la satisfaction des «besoins essentiels », mais encore faut-il que la croissance soit mise au service des besoins des individus. Or, si on se situe sur le versant des conditions de travail dans le monde, il y a loin de la coupe aux lèvres. Dans le $2^{\text {ème }}$ chapitre, l'auteur (qui est juriste) choisit un angle de discussion qui se situe sur le registre réglementaire et sur les rapports internationaux. En 1998, l'Organisation Internationale du Travail a défini des «normes fondamentales du travail»: le droit de négociation collective, l'élimination du travail forcé, l'abolition du travail des enfants et l'élimination de la discrimination. Un an plus tard, c'est la notion de «travail décent» qui sera promue au sein de l'OIT : le respect des normes fondamentales du travail, l'accès à la protection sociale, le dialogue social et l'accès à un emploi décent (le droit au travail). Cette notion de travail décent sera reprise en 2007 au sein de l'ONU. Mais ces normes sont loin d'être respectées.

Pourtant, la mise en œuvre d'un tel programme n'est nullement incompatible avec le développement économique (la croissance). Bazillier cherche à montrer ( $3^{\text {ème }}$ chapitre) que l'accès à l'emploi n'est pas opposé à la croissance; mais constitue au contraire une source d'efficacité économique. Le respect des règles internationales est un «investissement social » (pp. 68-74). Ainsi de la diminution du travail des enfants : ceci se traduit par un coût à court terme (coût d'éducation des enfants et effet d'opportunité du fait de salaires plus élevés), mais se traduit par une diminution du coût sur la collectivité (santé, traitement social) et d'un bénéfice à long terme lié à une meilleure productivité (du fait de la formation) et de meilleurs salaires (qui supportent la consommation intérieure).

C'est dans ce contexte que Bazillier attire l'attention sur la responsabilité sociale des entreprises. Pour l'auteur, la responsabilité sociale des entreprises résulte d'une pression de la société civile sur les entreprises, qui pallient alors aux régulations internationales. On tiendrait alors là un principe du développement durable appliqué à l'entreprise dont l'auteur tend à faire la promotion. Il souligne cependant deux limites. La première réside dans l'hétérogénéité et dans les difficultés d'évaluation des politiques réellement mises en œuvre (ce qui conduit à souhaiter une normalisation ou une procédure de qualification des telles 
entreprises). La seconde, d'inspiration régulationniste, conduit à argumenter que la responsabilité des entreprises est fondamentalement limitée : elles ne peuvent compenser complètement les défaillances des états, dès lors que personne n'impose le respect des chartes internationales.

Le $4^{\text {ème }}$ chapitre (travail et environnement) est consacré aux liens environnement - travail. Bazillier passe d'abord en revue (essentiellement à partir des travaux du GIEC) les risques de l'impact de la variable environnementale sur l'emploi. L'environnement est un facteur de production (ressources naturelles) nécessaire à la production. Si cette ressource diminue (disparition de la ressource) ou est altérée (pollution) l'impact économique est patent. D'autre part, l'environnement est susceptible d'avoir un impact sur les normes fondamentales de travail. À partir de là, Bazillier envisage la nécessaire adaptation de l'économie : par la mise en place de politique de juste répartition des conséquences, et par la recherche d'une économie verte dans laquelle la gestion de l'environnement pourrait être un facteur créateur d'emploi. Cette section est me semble-t-elle la plus limitée pour deux raisons : elle est quasi exclusivement centrée sur l'emploi (plutôt que sur le travail); et la variable environnementale y est essentiellement appréhendée sous le versant du réchauffement climatique (alors qu'elle concerne bien d'autres facteurs tels que gestion de l'eau, biodiversité, pression sur les ressources non renouvelables...).

La seconde section (intitulée « Acteur ») comporte deux parties.

- La première dresse une liste (internationale) de personnalités ayant œuvré au développement social en distinguant 5 catégories: les institutionnels et les politiques, syndicalistes, chefs d'entreprises et représentants patronaux, militants associatifs, et chercheurs.

- La seconde fait un inventaire des organisations impliquées dans la question du « travail décent » : organisations internationales, salariés, entreprises et représentants d'employeurs, ONG et la société civile.

Cette seconde section est très restreinte, et les critères de choix sont peu justifiés.

L'apport scientifique de cet ouvrage apparaîtra limité pour des ergonomes ou des psychologues du travail: malgré le titre, le travail reste une boîte noire (la focale est essentiellement l'emploi). Par ailleurs, l'auteur ne justifie pas toujours ses choix (tels que la centration exclusive sur le réchauffement climatique pour caractériser la variable environnementale). Mais il s'agit avant tout d'un ouvrage de vulgarisation, facile à lire et qui a le mérite de se positionner sur une thématique en attente de conceptualisations et de débats. À ce titre, cet ouvrage fournit une contribution dont on prend utilement connaissance. 